\title{
Regeneration of ischemic cardiac muscle and vascular endothelium by adult stem cells
}

\author{
Kathyjo A. Jackson, ${ }^{1}$ Susan M. Majka, ${ }^{1,2,3}$ Hongyu Wang, ${ }^{1}$ Jennifer Pocius, ${ }^{4}$ \\ Craig J. Hartley, ${ }^{4}$ Mark W. Majesky, ${ }^{3,5}$ Mark L. Entman, ${ }^{4}$ Lloyd H. Michael, ${ }^{4}$ \\ Karen K. Hirschi, ${ }^{1,2,3}$ and Margaret A. Goodell ${ }^{1}$
}

${ }^{1}$ Center for Cell and Gene Therapy and Department of Pediatrics,
${ }^{2}$ Children's Nutrition Research Center,
${ }^{3}$ Department of Molecular and Cellular Biology,
${ }^{4}$ DeBakey Heart Center, Methodist Hospital, and
${ }^{5}$ Department of Pathology, Baylor College of Medicine, Houston, Texas, USA

Address correspondence to: Margaret A. Goodell, Center for Cell and Gene Therapy, Baylor College of Medicine, N1030, One Baylor Plaza, Houston, Texas 77030, USA. Phone: (713) 798-1269; Fax: (713) 798-1230; E-mail: Goodell@bcm.tmc.edu. Kathyjo A. Jackson and Susan M. Majka contributed equally to this work.

Received for publication January 5, 2001, and accepted in revised form April 30, 2001.

\begin{abstract}
Myocyte loss in the ischemically injured mammalian heart often leads to irreversible deficits in cardiac function. To identify a source of stem cells capable of restoring damaged cardiac tissue, we transplanted highly enriched hematopoietic stem cells, the so-called side population (SP) cells, into lethally irradiated mice subsequently rendered ischemic by coronary artery occlusion for $60 \mathrm{~min}$ utes followed by reperfusion. The engrafted SP cells (CD34-/low, c-Kit $\left.{ }^{+}, \mathrm{Sca}^{-1}{ }^{+}\right)$or their progeny migrated into ischemic cardiac muscle and blood vessels, differentiated to cardiomyocytes and endothelial cells, and contributed to the formation of functional tissue. SP cells were purified from Rosa26 transgenic mice, which express lac $Z$ widely. Donor-derived cardiomyocytes were found primarily in the peri-infarct region at a prevalence of around $0.02 \%$ and were identified by expression of lacZ and $\alpha$-actinin, and lack of expression of CD45. Donor-derived endothelial cells were identified by expression of lacZ and Flt-1, an endothelial marker shown to be absent on SP cells. Endothelial engraftment was found at a prevalence of around $3.3 \%$, primarily in small vessels adjacent to the infarct. Our results demonstrate the cardiomyogenic potential of hematopoietic stem cells and suggest a therapeutic strategy that eventually could benefit patients with myocardial infarction.
\end{abstract}

J. Clin. Invest. 107:1395-1402 (2001).

\section{Introduction}

Occlusion of a coronary vessel and the resultant myocardial ischemia rapidly results in myocardial necrosis followed by scar formation. When the ischemic myocardium is reperfused, there is a rapid onset of contraction band necrosis and an intense inflammatory cascade. Inflammatory cells, although potentially injurious, are critical to efficient repair of the ventricular wall, mediating a process that involves resorption of necrotic material, scar formation, and angiogenesis (1). While efficient repair will allow the ventricle to function despite the loss of some of its cardiac myocytes (contractile subunits), it has been well established that adult cardiac myocytes do not replicate, thus these pump units are not actually replaced. Thus, microvascular repair can ensue through replication of smooth muscle cells and endothelial cells, but the ventricular myocytes are replaced by scar. The recent progress in the area of stem cell research has led to the suggestion that primitive stem cells might potentially be used to regenerate cells in organs in which no parenchymal regeneration occurs. The purpose of this study was to examine the possibility that primitive stem cells might contribute to regeneration in the infarcted myocardium.

Recent research suggests that primitive stem cells within whole bone marrow possess greater functional plasticity than was suspected previously. After bone marrow transplantation, donor-derived stem cells have been found in such diverse nonhematopoietic tissues as skeletal muscle (2), cardiac muscle (3), liver bile ducts $(4,5)$, and vascular endothelium (6-9). The stem cell compartment in human bone marrow is highly complex, comprising both $\mathrm{CD} 34^{+}$and CD34- hematopoietic stem cells, mesenchymal progenitors, and perhaps other cell types whose activities remain to be defined. In the study reported here, we tested a novel "side population" (SP) of CD34stem cells, selected on the basis of Hoechst dye staining, for their capacity to regenerate cardiac myofibers and blood vessels in ischemically injured cardiac tissue. SP cells have been well characterized previously and shown to be potent hematopoietic stem cells $(10,11)$ capable of contributing to regeneration of skeletal muscle (12). In the present study, SP cells have been more thoroughly characterized by RT-PCR and Ab staining in order to bet- 
ter define the differentiation potential of these transplanted stem cells. After their engraftment in the ischemically injured hosts, SP cells or their progeny became incorporated into both cardiac muscle and vessel structures, where they displayed the characteristics of differentiated cardiomyocytes as well as endothelial cells.

\section{Methods}

Isolation of SP cells. Bone marrow specimens extracted from the tibias and femurs of C57BL/6-Rosa (a mixture of homozygous and heterozygous animals) and C57BL/6-Ly-5.1 mice 6 to 12 weeks of age were suspended at $10^{6}$ cells $/ \mathrm{ml}$ in DMEM supplemented with 2\% FCS/10mM HEPES (HyClone Inc., Logan, Utah, USA, and Life Technologies Inc., Carlsbad, California, USA, respectively) and stained with $5 \mu \mathrm{g} / \mathrm{ml}$ Hoechst 33342 (Sigma-Aldrich, St. Louis, Missouri, USA) for 90 minutes at $37^{\circ} \mathrm{C}$, as described previously $(10,13)$. The cells were then resuspended in cold HBSS containing $2 \% \mathrm{FCS}$ and $2 \mu \mathrm{g} / \mathrm{ml}$ propidium iodide.

SP cells, selected as shown in Figure 1, typically accounted for $0.05 \%$ of whole bone marrow. These stem cells were additionally tested for c-Kit expression with an anti-c-Kit Ab (2B8; PharMingen, San Diego, California, USA) and for PECAM-1 (CD31) expression with anti-CD31-biotin (MEC 13,3; PharMingen) followed by staining with streptavidin-phycoerythrin (streptavidinPE) (Molecular Probes Inc., Eugene, Oregon, USA). Expression of the Tie- 2 protein was determined by Hoechst 33342 staining followed by fluorescein di- $\beta$-Dgalactopyranoside (FDG) staining (Molecular Probes Inc.) of whole bone marrow from mice expressing the lac $Z$ gene under control of the Tie-2 promoter, FVB/NTgN(TIE2LacZ)182 Sato (The Jackson Laboratory, Bar Harbor, Maine, USA) (14). Sorting and analysis of SP cells were performed on a triple-laser instrument (MoFlow; Cytomation Inc., Fort Collins, Colorado, USA). An argon laser tuned to 350-nm emission was used to excite the Hoechst dye. Fluorescence emission was collected with a 405/30 band pass (BP) filter (Hoechst blue) and a 670/40 BP filter (Hoechst red). A second 488-nm argon laser was used to excite PE and FITC. The purity of SP cells in the sorted samples was routinely greater than $91 \%$.

RNA extraction and RT-PCR analysis. Total RNA was extracted from purified SP cells from C57BL/6-Ly-5.1 mice using Tri-Reagent (Sigma-Aldrich). Approximately, $100 \mathrm{ng}$ of total RNA was treated with DNAase (Life Technologies Inc.) and used in a firststrand reaction that included oligo $\mathrm{dT}$ primers and Superscript reverse transcriptase (Life Technologies Inc.). Nested PCR was performed on cDNA under the following conditions: $94^{\circ} \mathrm{C} 1$ minute, $60^{\circ} \mathrm{C} 1$ minute, $72^{\circ} \mathrm{C} 1$ minute for 35 cycles. Five microliters of the first PCR reaction was used as a template in the nested PCR reaction. The following primers were used: angiopoietin-1 (580 bp), 5'-CAGTGGCTGCAAAAACTTGA-3' forward, 5'-TCTGCACAGTCTCGAAATGG-3' reverse; angiopoietin-2 (666 bp), $5^{\prime}$-CACACTGACCTTCCCCAACT-3' forward, 5'-TGGT-
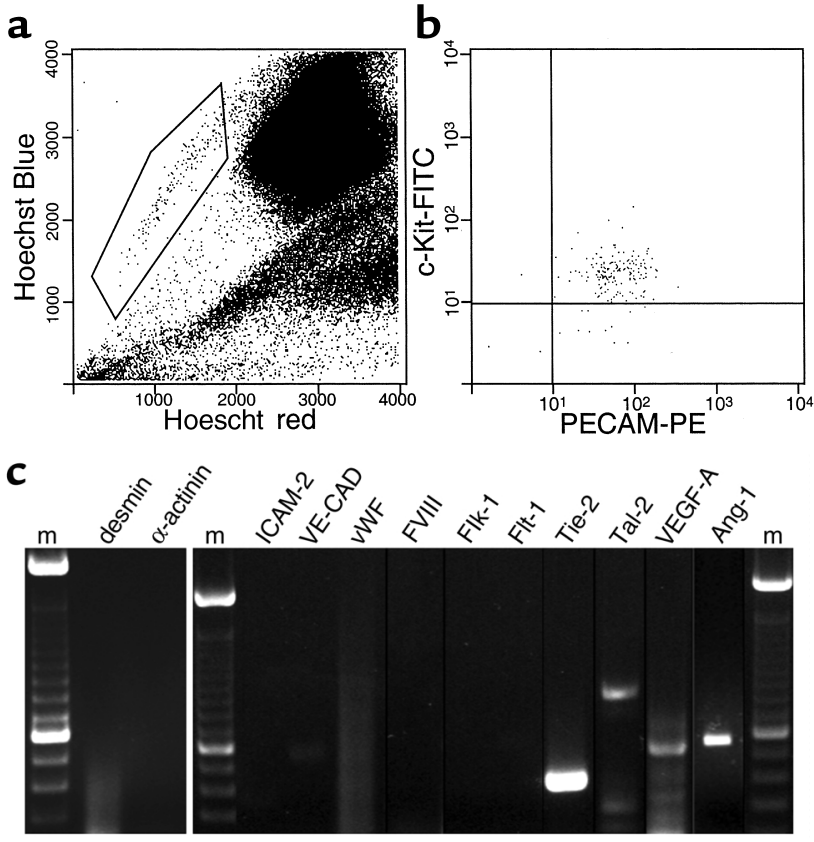

Figure 1

Gene-expression analysis of bone marrow SP cells. (a) Normal murine bone marrow is stained with Hoechst 33342. The indicated SP population comprises around $0.05 \%$ of total bone marrow cells. (b) The majority of SP cells are positive for the markers c-Kit and PECAM-1. (c) RT-PCR analysis of purified SP cells. m, marker; VE-CAD, VE-cadherin; FVIII, factor VIII.

GTCTCTCAGTGCCTTG-3' reverse; Tal-1 (984 bp), 5'GTCCTCACACCAAAGTAGTG-3' forward, 5'-AAACTAAGCAAGAATGAGATC- $3^{\prime}$ reverse; Tie-1 (300 bp), 5'-ACCCACTACCAGCTGGATGT-3' forward, 5'-ATCGTGTGCTAGCATTGAGG-3' reverse; Tie-2 (414 bp), 5'CCTTCCTACCTGCTA-3 $3^{\prime}$ forward-1, $5^{\prime}$-CCGTGGACAGGGGAGATAAT-3' forward-2, 5' ${ }^{\prime}$-CCACTACACCTTTCTTTACA-3' reverse; ICAM-2 (320 bp), $5^{\prime}$-CATATGGTCCGAGAAGCAGA-3' forward, 5' TGCACTCAATGGTGAAGTCT- $3^{\prime}$ reverse; VE-cadherin (520 bp), 5' -TTGCCCAGCCCTAGCAACCTAAAG-3' forward, 5'-ACCAC-CGCCCTCCTCATCGTAAGT-3' reverse; $v W F(1097 \mathrm{bp}), 5^{\prime}$-ATGATGGAGAGGTTACACATC forward-1, 5'-GCGCATCCGCGTGGCAGTGG-3' forward-2, 5'-GGCAGTTGCAGACCCTCCTTG-3' reverse; FVIII (400 bp), $5^{\prime}$-GTCCCTACTCCTTCTATTCTAGCC-3' forward-1, 5'-CTTCGCATGGAGTTGATGGGCTGT-3' forward-2, TCATCATAGGTGTGGATGAGTCCTG-3' reverse; $V E G F-$ $A(620,548,488$ bp), 5'-GGATCCATGAACTTTCTGCT$3^{\prime}$ forward-1, 5'-GGGTGCACTGGACCCTGGCT-3' forward-2, 5'-GAATTCACCGCCTCGGCTTGTC- $3^{\prime}$ reverse; Flk-1 (537 bp), 5'-GCCAATGAAGGGGAACTGAAGAC-3' forward, $5^{\prime}$-TCTGACTGCTGGTGATGCTGTC-3' reverse; Flt-1 (504 bp), 5'-TGTGGAGAAACTTGGTGACCT-3' forward, 5'-TGGAGAACAGCAGGACTCCTT-3' reverse; smooth muscle $\alpha$-actin (240 bp), $5^{\prime}$-GAGAAGCCCAGCCAGTCG-3' forward, 5'-CTCTTGCTCTGGGCTTCA-3' reverse; calponin (213 bp), $5^{\prime}$-CACCAACAAGTTTGCCAG-3' forward, 5' ${ }^{\prime}$-TGT- 
GTCGCAGTGTTCCAT-3' reverse; desmin (377 bp), 5'ATGAGCCAGGCCTACTCGTC- $3^{\prime}$ forward, $5^{\prime}$-GCGCACCTTCTCGATGTAGT- $3^{\prime}$ reverse; $\alpha$-actinin (976 bp), $5^{\prime}$-TGCTGCTATGGTGTCAGAGG-3' forward, 5'-CCGATCATTGACGTTCACAG- $3^{\prime}$ reverse.

Bone marrow transplantation and cardiac occlusion. Female C57BL/6-Ly-5.1 mice were irradiated (9 Gy) and injected with 2,000 SP cells isolated from male C57BL/6-Rosa-Ly-5.2 mice. Engraftment, which ranged from $35-85 \%$, was determined by analysis of peripheral blood using Abs against Ly-5.2 (clone 104; PharMingen) or FDG staining for lacZ expression (Molecular Probes Inc.) followed by FACS analysis. Approximately 10 weeks after bone marrow transplantation, the left anterior descending coronary artery of each mouse was occluded for 60 minutes, followed by reperfusion as described previously (15). Two or four weeks after cardiac injury, hearts were removed and frozen for immunohistochemical analysis. At 2 and 4 weeks, the survival rate was $26 \%$. Fifty-three percent of the mice survived at least 1 week, and $80 \%$ survived at least 24 hours of reperfusion after the 1 hour occlusion, consistent with previous observations.

Immunohistochemical analysis. Frozen sections $(12 \mu \mathrm{M})$ of experimental tissue were fixed with $4 \%$ paraformaldehyde for 30 minutes at $4^{\circ} \mathrm{C}$. LacZ staining was performed overnight at $37^{\circ} \mathrm{C}$ with the following reagents: $5 \mathrm{mM}$ potassium ferrocyanide, $5 \mathrm{mM}$ potassium ferricyanide, $2 \mathrm{mM} \mathrm{MgCl} 2,0.01 \%$ sodium deoxycholate, $0.02 \%$ Nonidet P-40 (NP-40), and 1 $\mathrm{mg} / \mathrm{ml} \mathrm{X-gal} \mathrm{(Life} \mathrm{Technologies} \mathrm{Inc.).} \mathrm{The} \mathrm{tissues} \mathrm{were}$ then washed in PBS and stained with specific Ab's. Anti- $\alpha$-actinin (clone EA-53; Sigma-Aldrich) was used to identify cardiac muscle, while anti-Flt-1 (clone C-17; Santa Cruz Biotechnology Inc., Santa Cruz, California, USA), and anti-ICAM-1 (generously provided by Alan Burns, Baylor College of Medicine) were used to recognize endothelial cells. Hematopoietic cells were visualized with anti-Ly-5-biotin (clone 30-F11; PharMingen). Secondary Ab's were conjugated to Alexa 488, Alexa 546, or Alexa 594 (all from Molecular Probes Inc.). Slides of the stained tissues were prepared and analyzed by fluorescence and differential interference contrast microscopy. Some sections were costained with the antimacrophage Ab, F480 (Serotec Ltd., Oxford, United Kingdom), which was detected with a kit from Vector Laboratories (Burlingame, California, USA) comprising a stain with a biotinylated anti-rat secondary followed by an avidin-biotin complex developed with 3,3-diaminobenzidine (DAB) from Vector Labs; the counterstain was eosin.

\section{Results}

The SP of murine bone marrow stem cells selected by Hoechst dye staining (Figure 1a) is a highly enriched hematopoietic stem cell population that can give rise to all hematopoietic lineages in the mouse $(10,11)$. To demonstrate a contribution from SP cells to the repair of nonhematopoietic tissues, we first tested for the expression of markers of primitive and fully differentiated endothelial cells and cardiomyocytes. As shown in Figure 1b, the intrinsically c-Kit-positive SP cells also expressed PECAM-1 (CD31), which was thought previously to be restricted to angioblasts, endothelial cells, megakaryocytes, and platelets. RT-PCR analysis of RNA from purified SP cells (Figure 1c) failed to detect common markers of cardiac muscle (desmin and $\alpha$-actinin), differentiated vascular endothelial cells (ICAM-2, VE-cadherin, $v W F$, and factor VIII), and early endothelial progenitor cells (Flk-1 and Flt-1, the receptors for VEGF). However, the SP cells did express the Tie-2 gene, which encodes a receptor for angiopoietins 1 and 2 . This finding was confirmed by examining the expression of lac $Z$ in SP cells from a mouse that expressed this gene under control of the Tie-2 promoter/enhancer (data not shown) $(14,16)$. The purified SP cells also expressed the early hematopoietic/endothelial cell transcription factor Tal$1 / S C L$, as well as three isoforms of VEGF-A and angiopoietin-1 (Ang-1). In all instances of marker positivity, the RT-PCR signal was present in three of three individual preparations of purified SP cells. The identities of the PCR products were confirmed by DNA sequencing.

To assess the contribution of SP cells to the repair of injured heart muscle, we employed bone marrow transplantation and a model of cardiac occlusion/reperfusion (Figure 2). Twenty-two C57Bl/6 mice were lethally irradiated and transplanted with SP cells purified from the bone marrow of 6-to-8-week-old adult $\mathrm{C} 57 \mathrm{Bl} / 6$ Rosa26 mice, a strain in which the lac $Z$ gene is expressed widely (17). Ten to 12 weeks after transplantation, the

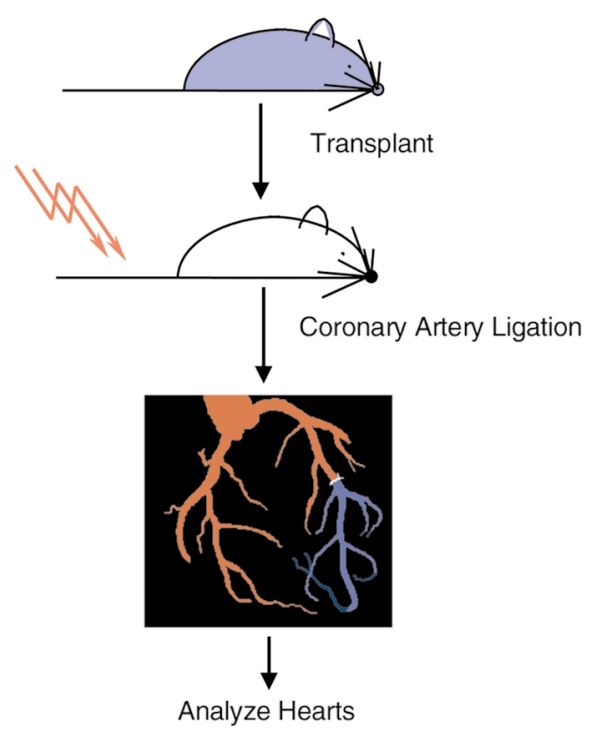

\section{Figure 2}

Experimental schema. SP cells were purified from bone marrow (BM-SP) obtained from C57Bl/6-Rosa26 transgenic mice. These cells were transplanted into lethally irradiated recipients. After 2 months, stable engraftment was determined by establishing the presence of lacZ-positive peripheral blood cells. Highly engrafted animals were subjected to coronary artery ligation for 60 minutes, followed by reperfusion. Two to four weeks later, the surviving animals were sacrificed for analysis of lacZ incorporation into cardiac tissue. 


\section{Figure 3}

Incorporation of SP cells into vascular endothelial cells. (a-d) X-gal-stained section of cardiac tissue from an infarcted SP cell-transplant recipient; $\mathbf{b}$ and $\mathbf{d}$ show magnifications of the indicated capillaries from $\mathbf{a}$ and $\mathbf{c}$. (e-I) Cardiac tissue as above, stained for expression of endothelial markers. An enlargement of the vessel stained for lacZ in $\mathbf{e}$ is seen in $\mathbf{f}$. The same vessel is shown in $\mathbf{g}$, stained with Flt- 1 , and in $\mathbf{h}$, with ICAM-1. An enlargement of the vessel stained for lac $\mathbf{Z}$ in $\mathbf{i}$ is seen in $\mathbf{j}$. The same vessel is shown in $\mathbf{k}$, stained for Flt 1 , and in $\mathbf{~}$, for ICAM1. a, c, e, and i: $\times 200 ; \mathbf{b}, \mathbf{d}, \mathbf{f}-\mathbf{h}$, and $\mathbf{j}-\mathbf{I}: \times 1,000$.

left anterior descending coronary artery was occluded for 1 hour and reperfused. Nineteen mice were treated, and sham operations were performed on three animals. Mice were sacrificed 2 or 4 weeks after injury in order to analyze hearts for incorporation of SP cells or their progeny into regenerating tissues. Five mice $(26 \%)$ that underwent occlusion/reperfusion of the coronary artery survived to the end of the experiment, a mortality rate consistent with previous observations (15).

The hearts of mice that survived for 2 or 4 weeks after injury were sectioned, stained with X-gal, followed by fluorescent Ab's, and scanned for incorporation of transplanted cells into regenerating tissues. We observed lac $Z$ staining in vessel structures of various caliber, but most commonly in capillaries (Figure 3, a-d). The lacZ-positive cells costained with Ab's against Flt-1 and ICAM-1 (Figure 3, e-1), demonstrating that SP cells or their progeny had migrated to the injured heart via the circulation, localized to newly forming vessels, and appeared to have integrated into the surface lining as differentiated endothelial cells. Analyses of SP-cell incorporation into specific vessel structures (i.e., arterioles, venules, lymphatic vessels) is ongoing. We are also further investigating the relative contribution of SP cells to the regeneration of specific vascular cell types (endothelial, pericyte, and smooth muscle cells). We found no lacZ-positive cells in vessel structures of sham-treated animals.

We also observed incorporation of lacZ-positive cells into cardiac muscle (Figure 4). In contrast to the sham-treated control, which lacked any evi-

\section{Figure 4}

Incorporation of SP cells into cardiomyocytes. (a) Negative control: C57BI/ 6 cardiac tissue stained for lacZ expression. (b) Positive control: C57Bl/6-Rosa26 cardiac tissue stained for lacZ expression. This typical section demonstrates both patterns of punctate and whole-fiber staining. (c) Cross-section of a heart from an SP cell-transplant recipient, which received an infarct. (d) Longitudinal section of an SP cell-transplant recipient, which received an infarct. (e-h) LacZ and $\alpha$-actinin costaining of lacZ-positive fibers. (i) CD45 costaining of the section in $\mathbf{g}$ and $\mathbf{h}$. (j) Anti-CD45 staining of spleen (positive control). Sections were stained with X-gal, and LacZ-positive sections were subsequently stained for $\alpha$-actinin $(\mathbf{f}, \mathbf{h})$ and CD45 (i), and the sections were photographed.

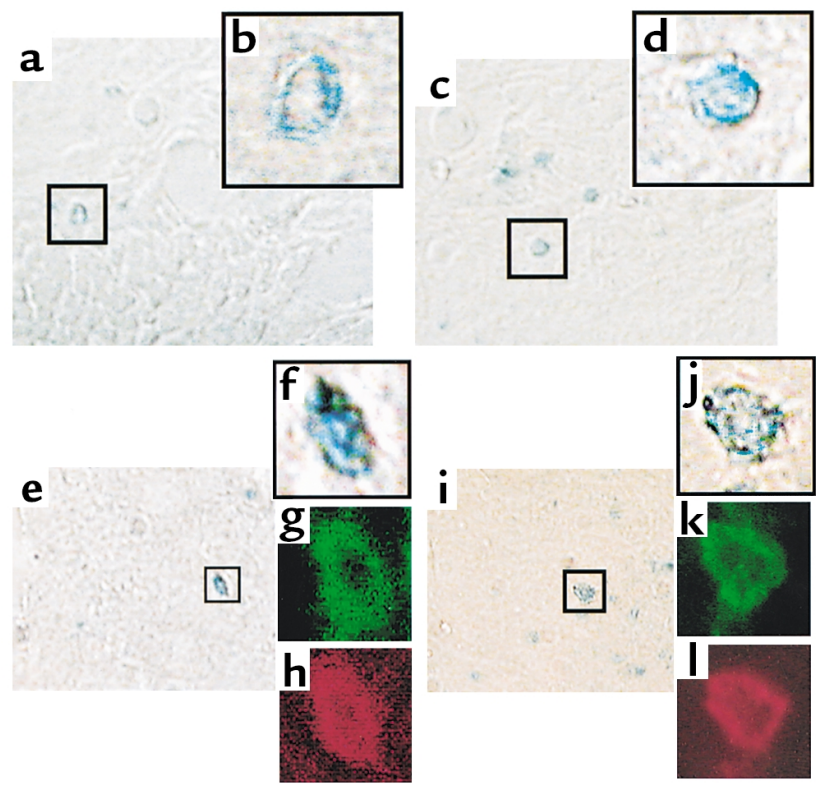

dence of SP-cell recruitment, cardiac tissue from the treated mice showed blue staining throughout the heart (Figure 4, c-h). Although the extent of staining varied appreciably, myocardial tissue specimens from all transplant recipients were lac $Z$ positive. In some cases, particularly in animal 6 , which had a remarkably high level of lacZ positivity, the staining extended throughout large tracts (Figure 4, c-e). In many cases, the staining was in a punctate pattern (Figure $4 \mathrm{~g}$ ). Cardiac muscle
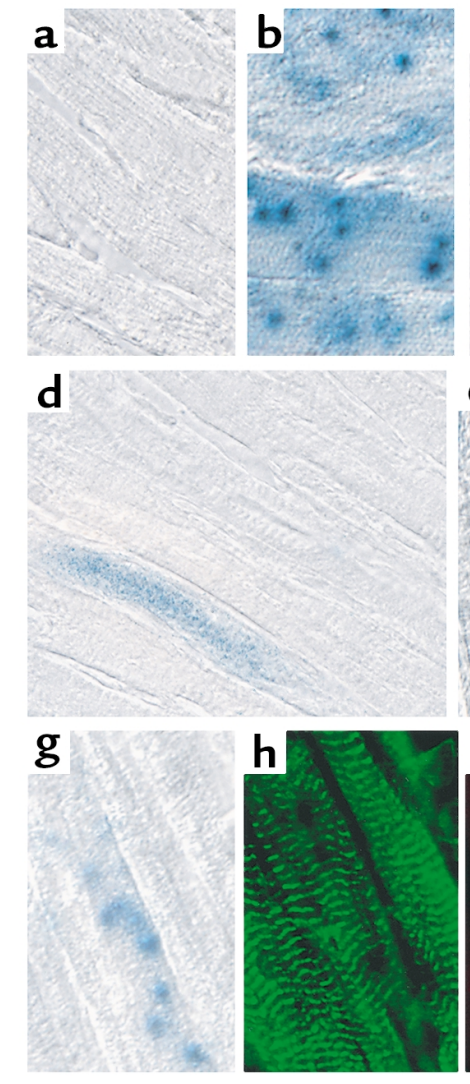
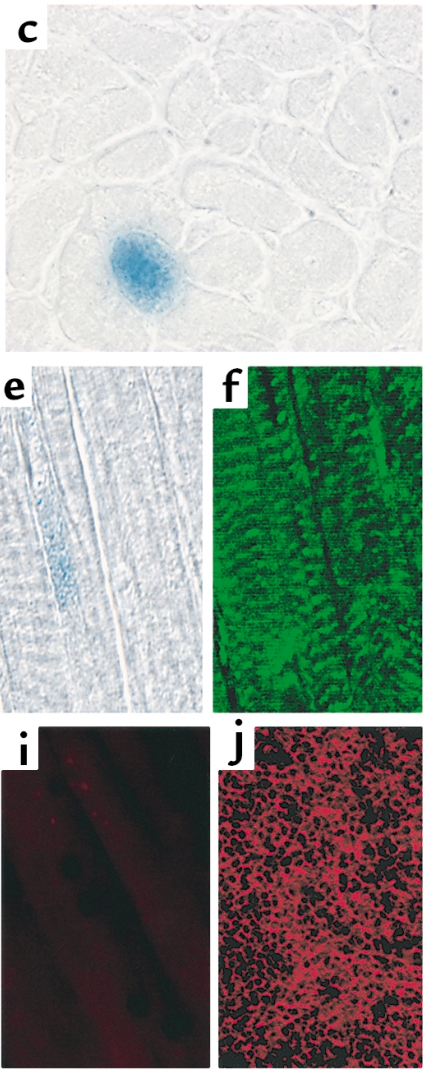


\section{Figure 5}

LacZ staining occurs primarily at the border of myocardial infarction. (a) Lower-power $(\times 10)$ photograph of mouse myocardial infarction after 4 weeks. The arrowhead points to the location of lacZ staining shown in $\mathbf{b}$ and $\mathbf{c}$. The lighter pink tissue to the left and above the arrowhead is primarily fibrotic and results from the infarction. (b) Higher-power $(\times 20)$ photograph of the same section dual stained for lacZ and the antimacrophage Ab F480. The open arrowhead indicates a macrophage, the closed arrowhead indicates lacZ-positive cardiomyocytes (the same region shown in Figure 4, $g$ and h). (c) Higher-power photograph of the same section $(\times 40)$. (d) Macrophage density of a cardiac section after 1 hour of ischemia and 3 hours of reperfusion. The open arrowheads indicate two of the many macrophages present. The counterstain is eosin.

from the Rosa 26 positive control stains in both a punctate and diffuse pattern (Figure $4 \mathrm{~b}$ ). The lacZpositive areas costained with Ab's against $\alpha$-actinin (Figure 4, e-h) and appeared to be contiguous with lacZ-negative cardiomyocytes. To be certain that the punctate staining pattern did not result from infiltrating hematopoietic cells, some sections were costained with Ab's against CD45, an antigen common to all nucleated hematopoietic cells (18). The lacZ-positive cells in Figure $4 \mathrm{~g}$ were not positive for CD45 (Figure 4i), although the CD45 Ab brightly stained spleen sections under the same conditions (Figure 4j). Only rarely were other lacZ-positive spots positive for CD45 (not shown).

LacZ-positive myocytes appeared primarily at the edge of the myocardial scar, corresponding to the region described as being "at risk" (15). This is shown in Figure 5, where lacZ-positive myocytes can be seen in a 4-week myocardial infarction. In this section, a macrophage can also be seen in the scar by costaining (Figure 5b, open arrowhead), but the lacZ-positive cells (closed arrowhead) do not stain. At 4 weeks, the macrophages have returned to a relatively low density; thus, the lac $Z$ staining seen in the myocyte is not explained by adhesive macrophages. Figure $5 \mathrm{~d}$ is included as a positive control to demonstrate, using the same $\mathrm{mAb}$, the macrophage density of a frozen section of a mouse heart after 1 hour of occlusion and 3 hours of reperfusion.

Table 1 shows the prevalence of lacZ-positive cells or structures in cardiac tissues from different mice. A total of four mice were examined in this experiment, three at 2 weeks after injury and one (the only surviving animal) at 4 weeks. Sections
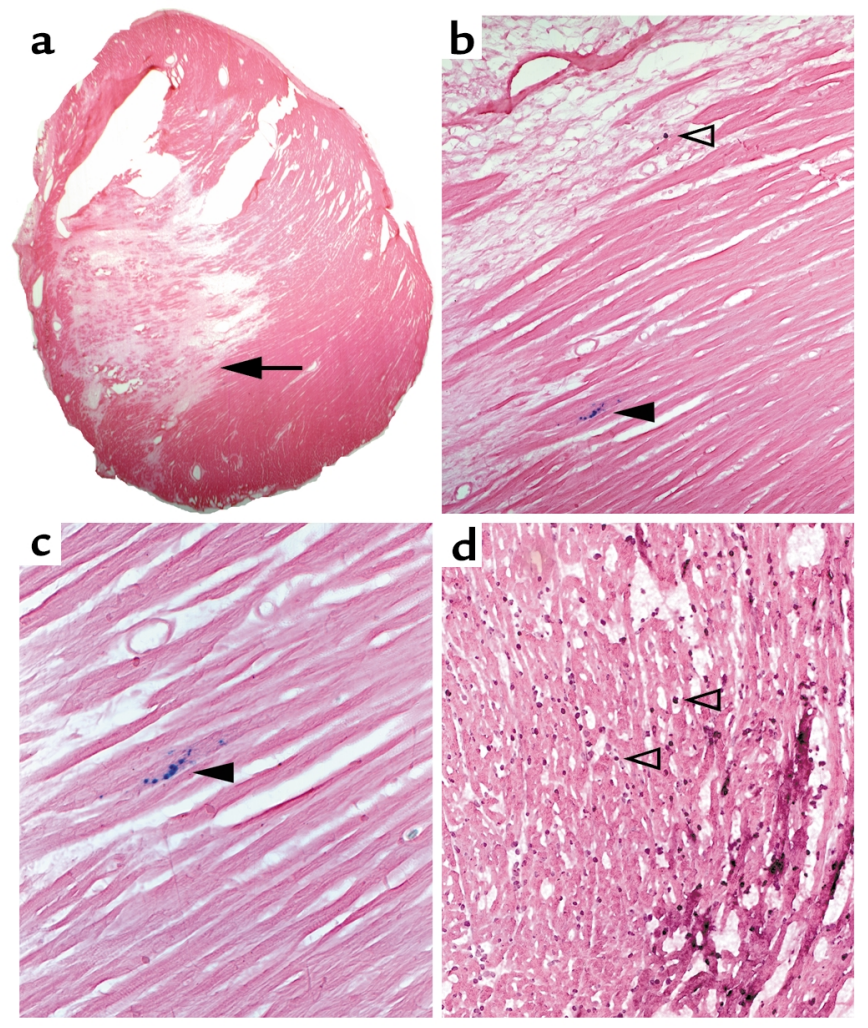

(100) from each animal were analyzed, and each positive site of lacZ staining was counted. Any staining pattern - a single blue cell, a cluster of blue cells, or an area of diffuse lacZ staining - was considered to represent one event of lacZ positivity or SP cell engraftment. The number of positive events ranged

\section{Table 1}

Prevalence of lacZ-positive cells and structures in cardiac tissues

\begin{tabular}{|c|c|c|c|c|c|}
\hline $\begin{array}{l}\text { Ischemic } \\
\text { mouse }\end{array}$ & $\begin{array}{c}\text { Week of } \\
\text { testing }\end{array}$ & $\begin{array}{l}\text { LacZ }^{+} \text {myofibers } \\
\text { per } 100 \text { sections }\end{array}$ & $\begin{array}{c}\% \text { cardiomyocyte } \\
\text { engraftment }\end{array}$ & $\begin{array}{c}\mathrm{LacZ}^{+} \text {vessels } \\
\text { (number per total) }\end{array}$ & $\begin{array}{c}\text { Mean \% endothelia } \\
\text { cell engraftment }\end{array}$ \\
\hline Sham & 4th & 0 & - & 0 & - \\
\hline No. 1 & $2 n d$ & 243 & 0.02 & $36 / 1100$ & $3.3 \pm 1.9$ \\
\hline No. 5 & 2nd & 55 & $<0.01$ & $11 / 600$ & $1.8 \pm 1.8$ \\
\hline No. 6 & 2nd & 730 & 0.06 & $39 / 1143$ & $3.7 \pm 2.5$ \\
\hline \multirow[t]{2}{*}{ No. 10} & 4th & 45 & $<0.01$ & $17 / 313$ & $5.4 \pm 0.9$ \\
\hline & & & Mean $0.02 \%$ & & Mean $3.3 \%$ \\
\hline
\end{tabular}

Animals were rendered ischemic by coronary artery ligation. Myofibers were counted as a single lacZ-positive structure, whether there was one blue cell, a cluster of blue cells, or a single large fiber stained throughout. The percentage of engraftment of cardiomyocytes is based on an estimate of 13,000 myocytes per section $(12,929 \pm 168 \mathrm{SD})$. This number was attained by photographing the total section of the mouse heart at the midventricular section used for all studies. On average, the sections contained $10.69 \mathrm{~mm}^{2}$ left-ventricular area; all sections were examined with little variance. Nuclei were counted in each section. The number of cardiac nuclei varied from 12 to $1,300 / \mathrm{mm}^{2}$. The average number of cardiac nuclei was taken as a reasonable estimate of the amount of cardiac myocytes visible within each section. The percentage of engraftment is based on counting 100 random sections of heart and therefore represents the engraftment for the entire cross-section, not just the area at risk. Since the peri-infarct region had a much higher prevalence of lacZ cardiomyocytes, the engraftment in the area at risk is probably larger, but the uncertainty of defining the area at risk on individual sections precluded a separate count. The vessels were quantified by determining the number of lacZ-positive vessel profiles (lacZ-positive vessel structures that were also costained with an Ab against Flt-1) per total vessels (Flt-1 positive) in the section as observed under the microscope. The mean percentage of lacZ-positive vessels is based on examination of 100 or more vessels of various sizes from 11 sections from mouse number 1 , six from mouse 5 , ten from mouse 6 , and three from mouse 10 . The vessels counted as positive had one or more endothelial cell that was lacZ positive and Flt-1 positive. 
from 45 to 730 per 100 sections. Since each section contained approximately 13,000 cardiomyocytes, this represents a mean engraftment prevalence of around $0.02 \%$ of all cardiomyocytes.

To determine incorporation of SP cells into endothelial cells, approximately 100 vessel profiles were analyzed per tissue section. Three to 11 tissue sections were counted per animal. As in the cardiac tissue, a single vessel was scored as positive whether one or multiple endothelial cells per vessel profile was stained. The mean prevalence of lacZ-positive vessels was approximately $3 \%$. This level of incorporation is somewhat lower than what has been reported for the engraftment of circulating endothelial-cell progenitors derived from whole blood (7). This is not surprising, considering that SP cells are multipotent and give rise to many cell types other than vascular endothelium. As expected, engraftment of the SP cells was predominantly into newly formed capillaries, in the "at-risk" tissue adjacent to the infarcted zone. Engraftment of SP cells into larger vessel structures was less frequent and to a lesser extent.

\section{Discussion}

Myocardial infarction is a leading cause of heart failure and death in developed countries. Although postinfarction survival rates have improved in recent years, reduced heart function due to excessive loss of cardiomyocytes remains a major problem. The lack of resident stem cells in the heart has led to an intensive search for alternative sources of cardiomyocyte progenitors. Embryonal stem cells have been shown to differentiate into cardiomyocytes that can form stable intracardiac grafts (19). Skeletal myoblasts or cardiomyocytes from fetal or neonatal mice have also been shown to take up residence in cardiac tissue after injury $(20,21)$. Although demonstrating the potential of cellular engraftment as a means to augment the number of myocytes in cardiac muscle, these previous studies have not identified a progenitor population readily accessible from an adult patient's own tissue that can be adapted to clinical therapy.

Here we have assessed the ability of purified stem cells derived from adult mouse bone marrow to participate in cardiac muscle regeneration following the induction of ischemia by coronary artery occlusion and reperfusion. These stem cells are a highly enriched stem cell population that can be reproducibly isolated from adult tissues. When transplanted into the bone marrow of lethally irradiated mice, purified SP cells marked with the lac $Z$ gene regenerated the hematopoietic system. At 2 or 4 weeks after coronary artery ligation, lacZpositive cells had migrated into cardiac myofibers, where they participated in the regeneration of healthy muscle. This level of myocardial engraftment, $0.02 \%$ of all cardiomyocytes, was similar to that seen with use of whole bone marrow or purified stem cells to regenerate skeletal muscle $(2,12)$. Importantly, there was no evidence of SP cell migration into the hearts of shamoperated animals. Finally, the presence of lacZ-positive hematopoietic cells in the cardiac tissue (measured with Ab's against CD45 and macrophages) was not a problem in this study, as shown in Figure 5. This is consistent with previous observations that the major inflammatory response is observed immediately after infarction and has largely resolved by 2 to 4 weeks (22).

We also found that lacZ-positive cells could participate in neovascularization in regenerating heart tissue. Many small vessels, comprising one to three endothelial cells (cell number determined by 4',6-diamidine-2phenylindole dihydrochloride [DAPI] staining), clearly expressed the marker gene. Some large- or mediumsized vessels also appeared to have incorporated marked cells, although such structures consisted primarily of host cells. The overall level of neovascular engraftment was approximately $3 \%$. This is lower than the engraftment of more differentiated endothelial cell progenitors derived from whole blood (7). The majority of engraftment also appeared to be adjacent to the region of infarct. We did not attempt to determine whether these vessel structures were arterioles, venules, or lymphatic vessels.

We present evidence here that bone marrow hematopoietic stem cells, purified as SP cells, have transdifferentiated and engrafted into myocardium and endothelial cells. However, future studies can extend and confirm these conclusions using additional criteria. First, we cannot exclude the possibility that small numbers of contaminating nonhematopoietic stem cells are responsible for the cardiac and endothelial cell engraftment. Such contaminants could arise from imperfect cell sorting or could even be due to stem cells copurified by our SP isolation procedure. Clonal studies will be essential to rule out the possibility that multiple stem cell populations are present within the donor cell population. Second, current studies in the field are limited by the use of markers that are present ubiquitously in donor cells, such as lac $Z$, green fluorescent protein (GFP), or Y chromosome fluorescence in situ hybridization (FISH) $(12,23)$. While one could argue about differences in sensitivity and specificity of these marker systems, each will identify any donor cell, not just one that has differentiated into the cell type of interest. This necessitates the use of secondary criteria, such as morphology and colocalization with specific markers, to establish cell identity. Perhaps more definitive will be using donor cells that express a nuclear-localized marker gene under the control of a cell type-specific promoter. In this case, donor cells will not express the marker until they differentiate into the cell type of interest (2). Although such studies should generate less disputable data, they are also flawed in that expression of any one gene, at any given time, is not a reliable indicator of a distinct phenotype. Hence, determining donor cell localization into complex tissues in this manner will still require colocalization with other markers, as well.

Our detection of angioblast markers on purified SP cells may bear on the developmental fate of these stem 
cells. Before transplantation took place, we observed no expression of genes indicative of a mature, differentiated endothelial cell phenotype in SP cells. Instead, our analyses revealed, as expected, expression of genes reported to be activated in a common progenitor of both hematopoietic cells and endothelial cells, namely PECAM-1 (24) and Tal-1 (25-27). Surprisingly, other genes thought to be specifically reflective of an embryonic endothelial cell progenitor, such as tyrosine kinase receptors Flk-1 and Flt-1 (28), were not expressed in SP cells; however, expression of their ligand VEGF-A was detected.

Perhaps expression of VEGF-A in the endogenous population of SP cells plays a role in "directing" these multipotent cells to the surface of mature endothelial cells expressing VEGF receptors in adult tissues when needed. Such a role for VEGF-A, via Flk-1-receptor signaling, has been described for angioblast migration during embryonic vascular development (29), supporting a role of VEGF-receptor binding in directing the positioning, as well as differentiation, of multipotent progenitors. Alternatively, it is also possible that VEGF-A expression by multipotent stem cells may serve a unique functional role in the stem cell microenvironment, independent of its well-documented effects on endothelial cell behavior (30), perhaps in the maintenance of a "progenitor" state.

In addition to expressing VEGF-A, the bone marrow SP cells also expressed RNA for Ang-1 and its receptor, Tie-2. Ang- 1 and Tie- 2 are thought to be involved in an important paracrine-signaling pathway needed for the recruitment, or maintenance, of smooth muscle cells and pericytes to stabilize newly forming endothelial tubes (31). Embryos lacking either Ang-1 (31) or Tie-2 (16) die at embryonic day 11.5 and 10.5 , respectively, with severe vascular malformations and hemorrhage. The role of Ang-1 and Tie-2 in the SP cells is uncertain. Since SP cells express the ligand, as well as the receptor, both autocrine and paracrine regulatory pathways can be envisioned. In a paracrine manner, perhaps Ang-1 or Tie- 2 bind to their respective partner in the bone marrow stroma to modulate cell survival or responses to other soluble factors in the microenvironment, as has been proposed for mature endothelial cells (32). Certainly, much remains to be determined regarding the role of factors such as Ang-1 and VEGF-A in the modulation of SP survival, growth control, and interactions with the surrounding microenvironment.

Although endothelial cells and hematopoietic cells are thought to be related developmentally, possibly originating from a common hemangioblast precursor (33), evidence for such a cell in the adult is lacking. We suggest that the SP cells are bona fide hematopoietic stem cells with the capacity for endothelial or cardiomyocytic differentiation under circumstances of acute injury. Our findings underscore the developmental versatility of adult hematopoietic stem cells and suggest that their functional role is ultimately determined by their migration into particular microenvi- ronments, such as myocardium, and their exposure to locally generated signals at those sites.

What cell type participates in myocardial regeneration? In our experiment, the purified stem cells stably engrafted into the bone marrow of recipient mice. The presence of lacZ-marked cells in damaged cardiac tissue could reflect direct incorporation of migrating SP cells or their progeny. Since the surface phenotypes of lac $Z$-positive cells that had integrated into cardiac tissues were those of differentiated myocytes and endothelial cells, we are unable to deduce the exact identity of the cells that first migrated into the heart.

The incorporation of bone marrow-derived SP cells into both regenerating endothelial cells and cardiomyocytes suggests that circulating stem cells may naturally contribute to repair of these tissues. The lack of sensitive markers and similar experimental design has likely impeded previous observation. Although the contribution of our stem cells to cardiac regeneration was low, improvements in efficiency that can be achieved through a better understanding of the process promise to offer new therapeutic avenues in the long term.

\section{Acknowledgments}

We thank Michael Cubbage and Brian Newsom for performing flow cytometry and John Gilbert and Alan Burns for comments on the manuscript. K.A. Jackson is a fellow of the Leukemia and Lymphoma Society. M.A. Goodell is an American Society of Hematology Fellow. This work was funded in part by grants to M.A. Goodell from the Muscular Dystrophy Association and the NIH; grants to K.K. Hirschi from the American Heart Association (SDG-9930054N), the USDA, the NIH (HL-61408), and the Gillson Longenbough Foundation; to M.L. Entman from the NIH (HL-42550), the DeBakey Heart Center at Baylor College of Medicine, and the Methodist Hospital; and to M.W. Majesky by the NIH (HL-47655). M.W. Majesky is an Established Investigator of the American Heart Association.

1. Entman, M.L., and Smith, C.W. 1994. Postreperfusion inflammation: a model for reaction to injury in cardiovascular disease. Cardiovasc. Res. 28:1301-1311.

2. Ferrari, G., et al. 1998. Muscle regeneration by bone marrow-derived myogenic progenitors. Science. 279:1528-1530.

3. Bittner, R.E., et al. 1999. Recruitment of bone-marrow-derived cells by skeletal and cardiac muscle in adult dystrophic mdx mice. Anat. Embryol. (Berl.) 199:391-396.

4. Lagasse, E., et al. 2000. Purified hematopoietic stem cells can differentiate into hepatocytes in vivo. Nat. Med. 6:1229-1234.

5. Petersen, B.E., et al. 1999. Bone marrow as a potential source of hepatic oval cells. Science. 284:1168-1170.

6. Asahara, T., et al. 1997. Isolation of putative progenitor endothelial cells for angiogenesis. Science. 275:964-967.

7. Schatteman, G.C., Hanlon, H.D., Jiao, C., Dodds, S.G., and Christy, B.A. 2000. Blood-derived angioblasts accelerate blood-flow restoration in diabetic mice. J. Clin. Invest. 106:571-578.

8. Takahashi, T., et al. 1999. Ischemia- and cytokine-induced mobilization of bone marrow-derived endothelial progenitor cells for neovascularization. Nat. Med. 5:434-438.

9. Shi, Q., et al. 1998. Evidence for circulating bone marrow-derived endothelial cells. Blood. 92:362-367.

10. Goodell, M., Brose, K., Paradis, G., Conner, A., and Mulligan, R. 1996. Isolation and functional properties of murine hematopoietic stem cells that are replicating in vivo. J. Exp. Med. 183:1797-1806. 
11. Goodell, M.A., et al. 1997. Dye efflux studies suggest the existence of CD34-negative/low hematopoietic stem cells in multiple species. Nat. Med. 3:1337-1345.

12. Gussoni, E., et al. 1999. Dystrophin expression in the $\mathrm{mdx}$ mouse restored by stem cell transplantation. Nature. 401:390-394.

13. Goodell, M.A. 1998. http://www.bcm.tmc.edu/genetherapy/ goodell/.

14. Schlaeger, T.M., et al. 1997. Uniform vascular-endothelial-cell-specific gene expression in both embryonic and adult transgenic mice. Proc. Natl. Acad. Sci. USA. 94:3058-3063.

15. Michael, L.H., et al. 1995. Myocardial ischemia and reperfusion: a murine model. Am. J. Physiol. 269:H2147-H2154.

16. Sato, T., et al. 1995. Distinct roles of the receptor tyrosine kinases Tie-1 and Tie-2 in blood vessel formation. Nature. 376:70-74.

17. Zambrowicz, B.P., et al. 1997. Disruption of overlapping transcripts in the ROSA $\beta$ geo 26 gene trap strain leads to widespread expression of $\beta$ galactosidase in mouse embryos and hematopoietic cells. Proc. Natl. Acad. Sci. USA. 94:3789-3794.

18. Terry, L.A., Brown, M.H., and Beverley, P.C. 1988. The monoclonal antibody, UCHL1, recognizes a 180,000 MW component of the human leucocyte-common antigen, CD45. Immunology. 64:331-336.

19. Klug, M.G., Soonpaa, M.H., Koh, G.Y., and Field, L.J. 1996. Genetically selected cardiomyocytes from differentiating embryonic stem cells form stable intracardiac grafts. J. Clin. Invest. 98:216-224.

20. Taylor, D.A., et al. 1998. Regenerating functional myocardium: improved performance after skeletal myoblast transplantation. Nat. Med. 4:929-933.

21. Reinecke, H., Zhang, M., Bartosek, T., and Murry, C.E. 1999. Survival, integration, and differentiation of cardiomyocyte grafts: a study in normal and injured rat hearts. Circulation. 100:193-202.

22. Frangogiannis, N.G., Michael, L.H., and Entman, M.L. 2000. Myofibroblasts in reperfused myocardial infarcts express the embryonic form of smooth muscle myosin heavy chain (SMemb). Cardiovasc. Res. 48:89-100.

23. Brazelton, T.R., Rossi, F.M., Keshet, G.I., and Blau, H.M. 2000. From marrow to brain: expression of neuronal phenotypes in adult mice. Science. 290:1775-1779.

24. Yamashita, J., et al. 2000. Flk1-positive cells derived from embryonic stem cells serve as vascular progenitors. Nature. 408:92-96.

25. Kallianpur, A.R., Jordan, J.E., and Brandt, S.J. 1994. The SCL/TAL-1 gene is expressed in progenitors of both the hematopoietic and vascular systems during embryogenesis. Blood. 83:1200-1208.

26. Gering, M., Rodaway, A.R., Gottgens, B., Patient, R.K., and Green, A.R 1998. The SCL gene specifies haemangioblast development from early mesoderm. EMBOJ. 17:4029-4045

27. Drake, C.J., Brandt, S.J., Trusk, T.C., and Little, C.D. 1997. TAL1/SCL is expressed in endothelial progenitor cells/angioblasts and defines a dorsal-to-ventral gradient of vasculogenesis. Dev. Biol. 192:17-30.

28. Drake, C.J., and Fleming, P.A. 2000. Vasculogenesis in the day 6.5 to 9.5 mouse embryo. Blood. 95:1671-1679.

29. Cleaver, O., and Krieg, P.A. 1998. VEGF mediates angioblast migration during development of the dorsal aorta in Xenopus. Development. 125:3905-3914.

30. Neufeld, G., Cohen, T., Gengrinovitch, S., and Poltorak, Z. 1999. Vascular endothelial growth factor (VEGF) and its receptors. FASEB J. 13:9-22.

31. Suri, C., et al. 1996. Requisite role of angiopoietin-1, a ligand for the TIE2 receptor, during embryonic angiogenesis. Cell. 87:1171-1180.

32. Papapetropoulos, A., et al. 1999. Direct actions of angiopoietin-1 on human endothelium: evidence for network stabilization, cell survival, and interaction with other angiogenic growth factors. Lab. Invest. 79:213-223.

33. Choi, K., Kennedy, M., Kazarov, A., Papadimitriou, J.C., and Keller, G. 1998. A common precursor for hematopoietic and endothelial cells. Development. 125:725-732. 